\title{
Optimal navigation strategies for microswimmers on curved manifolds
}

\author{
Lorenzo Piro $\odot,{ }^{1}$ Evelyn Tang $\odot,{ }^{1}$ and Ramin Golestanian $\odot^{1,2, *}$ \\ ${ }^{1}$ Max Planck Institute for Dynamics and Self-Organization (MPIDS), 37077 Göttingen, Germany \\ ${ }^{2}$ Rudolf Peierls Centre of Theoretical Physics, University of Oxford, Oxford OX1 3PU, United Kingdom
}

(Received 15 October 2020; revised 2 February 2021; accepted 29 April 2021; published 18 May 2021)

\begin{abstract}
Finding the fastest path to a desired destination is a vitally important task for microorganisms moving in a fluid flow. We study this problem by building an analytical formalism for overdamped microswimmers on curved manifolds and arbitrary flows. We show that the solution corresponds to the geodesics of a Randers metric, which is an asymmetric Finsler metric that reflects the irreversible character of the problem. Using the examples of spherical and toroidal surfaces, we demonstrate that the swimmer performance that follows this "Randers policy" always beats a more direct policy. Moreover, our results show that the relative gain grows significantly when specific structures related to either the geometry or the flow are exploited by the swimmer. A study of the shape of isochrones reveals features such as self-intersections, cusps, and abrupt nonlinear effects. Our work provides a link between microswimmer physics and geodesics in generalizations of general relativity.
\end{abstract}

DOI: 10.1103/PhysRevResearch.3.023125

\section{INTRODUCTION}

Artificial micro- and nanoswimmers [1] with active external controls (e.g., via chemical [2,3] and electromagnetic fields [4-6], feedback loops [7,8], and geometric features of boundaries [9]) can increasingly be engineered to execute specialized tasks in complex environments. These have crucial technological and medical applications ranging from targeted delivery of drugs [10], genes [11], or other cargo [12], to prevention of dental biofilm [13]. In addition, it is beneficial for microorganisms such as bacteria, algae, or spermatozoa to employ sensing mechanisms equipped with adaptation strategies to control their motility machinery in order to find the fastest path towards a desired destination [14], e.g., when tracking a food source [15] or seeking light [16]. Such navigation typically takes place in the presence of a fluid flow or an external force landscape, which can hinder or help their motion. The optimal path is hence distinct from the shortest path, rendering this a complex problem in the field of active matter [17].

Optimal navigation was first addressed by Zermelo, who studied a ship navigating in the presence of an external wind [18]. Recent work has explored this problem for microorganisms navigating on two-dimensional surfaces and shown exact results for some classes of force fields (linear, shear, or vortex fields) [19]. Other approaches include algorithmic optimization procedures based on the application of machine

\footnotetext{
*ramin.golestanian@ds.mpg.de
}

Published by the American Physical Society under the terms of the Creative Commons Attribution 4.0 International license. Further distribution of this work must maintain attribution to the author(s) and the published article's title, journal citation, and DOI. Open access publication funded by the Max Planck Society. learning to active motion [20-22]. Motion on curved surfaces and the role of local geometry have recently gained significant interest in the field of active matter [23] (e.g., in cells and tissue organization [24], cell motility [25], or collective motion of active particles [26,27]). Here, we aim to develop an analytical formalism for optimal navigation in an overdamped system, which can be used on curved manifolds and arbitrary stationary flows.

Adopting recent mathematical results from differential geometry [28,29], we show that this problem can be mapped onto geodesics of a Finsler-type geometry with a Randers metric [30]. Finsler spaces are more general than Riemannian spaces since the tangent norms need not be induced by inner products [31]. They have been used to construct geometric descriptions in many areas of physics, with applications ranging from electron motion in magnetic flows [32] to quantum control [33] and test theories of relativity [34]. The particular choice of the asymmetric Randers metric allows us to characterize the irreversibility of the optimal trajectory in this nonequilibrium problem.

We start by illustrating the formalism and discussing some general properties of the system. Then, we apply these concepts to some specific setups and study how following Randers geodesics can reduce the travel time to reach a target compared to when the microswimmer heads constantly towards it. Lastly, we analyze the isochrones - curves of equal travel time- to investigate more generally the shape of optimal paths.

\section{CURVED MANIFOLDS AND FINSLER GEOMETRY}

Consider a microswimmer that is free to move on a smooth Riemannian manifold $\mathcal{M}$ [see Fig. 1(a)] equipped with a positive definite metric $h$, such that the corresponding norm of any tangent vector $\boldsymbol{x} \in T \mathcal{M}$ can be calculated via $|\boldsymbol{x}|_{h}^{2}=$ $h_{i j} x^{i} x^{j}$, where Einstein's summation convention is used [35]. 

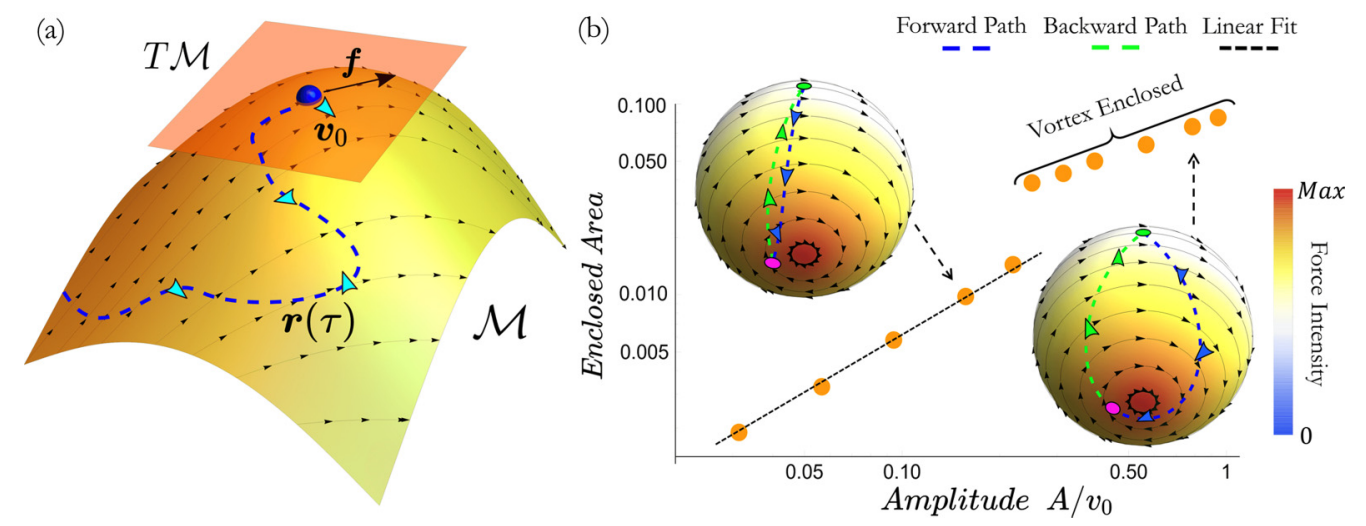

FIG. 1. (a) Microswimmer trajectory $\boldsymbol{r}(\tau)$ (blue dashed line) on a Riemannian manifold $\mathcal{M}$. The microswimmer moves in the tangent space $T \mathcal{M}$ under the joint influence of the force $\boldsymbol{f}$ (black arrows) and its self-propelling velocity $\boldsymbol{v}_{0}$, which is marked with cyan arrows. (b) Fraction of a spherical surface enclosed in the optimal forward-backward loop as a function of the force amplitude. The plot is in log-log scale. There is a jump when the vortex is enclosed in the loop. This introduces a qualitative difference which is reflected in the change from linear to sublinear scaling. There are two exemplar trajectories for given values of the force amplitude as indicated by dashed black arrows. The forward (blue dashed) and backward (green dashed) paths connect the following two points: $\left(\theta_{0}, \phi_{0}\right)=\left(\frac{\pi}{2}, 0\right) \rightleftarrows\left(\theta_{1}, \phi_{1}\right)=\left(\frac{11 \pi}{12}, \frac{14 \pi}{10}\right)$. The arrows on top of each trajectory indicate the heading direction of the microswimmer. The intensity (color) gradient on each sphere shows the force intensity, while the solid black arrows show the force direction.

The Riemannian metric can also be used to define the scalar product of any two tangent vectors $\boldsymbol{x}, \boldsymbol{y} \in T \mathcal{M}$ as $h_{i j} x^{i} y^{j}$. Let us now assume that the microswimmer moves on such a surface with the self-propulsion velocity $\boldsymbol{v}_{0}$, which corresponds to a constant speed $\left|\boldsymbol{v}_{0}\right|_{h} \equiv v_{0}$. The motion takes place in the presence of a time-independent force field $\boldsymbol{f}(\boldsymbol{r})$, which may in general include a contribution due to advection by the solvent flow velocity (note that the friction coefficient is set to unity). The overdamped motion of the microswimmer can therefore be described as follows:

$$
\frac{d \boldsymbol{r}}{d \tau}=\boldsymbol{v}_{0}+\boldsymbol{f}[\boldsymbol{r}(\tau)]
$$

where $\tau$ is the swimmer ("proper") time [see Fig. 1(a)]. We neglect rotational noise and assume full control over the direction of microswimmer propulsion as described by $\boldsymbol{v}_{0}$. This means that the direction of propulsion is steered by a protocol that selects the appropriate active angular velocity to make it follow a prescribed path.

To show how Finsler geometry enters the optimal navigation problem on curved manifolds, we consider the time for a microswimmer to go from one point $\boldsymbol{r}_{\mathrm{A}}$ to another $\boldsymbol{r}_{\mathrm{B}}$ on the surface via the trajectory $\boldsymbol{r}(s)$ that is parametrized with $s$ :

$$
T=\int_{r_{\mathrm{A}}}^{r_{\mathrm{B}}} d \tau=\int_{r_{\mathrm{A}}}^{r_{\mathrm{B}}} \frac{d s}{v} \equiv \int_{r_{\mathrm{A}}}^{r_{\mathrm{B}}} d s \mathcal{L}[s, \boldsymbol{r}(s), \dot{\boldsymbol{r}}(s)],
$$

where $v \equiv \frac{d s}{d \tau}, \dot{\boldsymbol{r}} \equiv \frac{d \boldsymbol{r}}{d s}$, and the Lagrangian $\mathcal{L} \equiv v^{-1}$ is defined by identifying the traveling time as an action. Using Eq. (1), we obtain the following expression for the Lagrangian (see Appendix A):

$$
\mathcal{L}=\sqrt{a_{i j} \dot{r}^{i} \dot{r}^{j}}+b_{i} \dot{r}^{i},
$$

where we have used the definitions $a_{i j} \equiv h_{i j} \lambda+f_{i} f_{j} \lambda^{2}, b_{i} \equiv$ $-f_{i} \lambda$, and $\lambda^{-1} \equiv v_{0}^{2}-h_{i j} f^{i} f^{j}$, with $f_{i}=h_{i j} f^{j}$. We now make the observation that the resulting Lagrangian has all the defining features to be a Finsler metric of Randers type [30], if and only if the condition $|\boldsymbol{f}|_{h}<v_{0}$ is fulfilled at any point on the surface and any time (see Ref. [29] for a proof). This implies that the self-propulsion is assumed to be able to overpower the external force $\boldsymbol{f}$ at all points. Such a constraint ensures that $\mathcal{L}$ is strongly convex and positive definite, two necessary features for identification as a Randers metric [36-38].

\section{RANDERS SPACES AND IRREVERSIBILITY}

Randers spaces are often referred to as a special class of nonreversible Finsler spaces [36]. This is due to the presence of the second term in (3), namely, $b_{i} \dot{r}^{i}$, which makes the metric tensor manifestly asymmetric under time reversal, i.e., $\mathcal{L}\left(\dot{r}^{i}\right) \neq \mathcal{L}\left(-\dot{r}^{i}\right)$. Due to this asymmetry, in the presence of an external force the optimal forward path (between $\boldsymbol{r}_{\mathrm{A}}$ and $\left.\boldsymbol{r}_{\mathrm{B}}\right)$ will in general be different from the backward one $\left(\boldsymbol{r}_{\mathrm{B}}\right.$ to $\boldsymbol{r}_{\mathrm{A}}$ ). In other words, the optimal backward path is distinct from the time-reversed forward path, which highlights the outof-equilibrium character of the navigation problem we study. In contrast, Riemannian geodesics (in the absence of any external force) are reversible since the corresponding metric tensor $h$ is symmetric [39]. This property of Randers metrics is illustrated with a concrete example in Fig. 1(b) and studied in more detail below.

Since $\mathcal{L}$ is a homogeneous function of degree one with respect to $\dot{r}^{i}$, we can introduce the fundamental tensor

$$
g_{i j} \equiv \frac{1}{2} \frac{\partial^{2} \mathcal{L}^{2}}{\partial \dot{r}^{i} \partial \dot{r}^{j}},
$$

which is also positive definite due to the convexity condition [38]. For the Randers metric of Eq. (3), we find

$$
g_{i j}=\left(1+\frac{b_{i} \dot{r}^{i}}{\sqrt{a_{i j} \dot{r}^{i} \dot{r}^{j}}}\right)\left(a_{i j}-\ell_{i} \ell_{j}\right)+\left(b_{i}+\ell_{i}\right)\left(b_{j}+\ell_{j}\right),
$$

where $\ell_{i} \equiv a_{i j} \dot{r}^{j} / \sqrt{a_{i j} \dot{r}^{i} \dot{r}^{j}}$. In order to determine the timeminimizing paths, we solve the Euler-Lagrange equations for the corresponding energy functional $\mathcal{E}=\frac{1}{2} \mathcal{L}^{2}=g_{i j} \dot{r}^{i} \dot{r}^{j}$, namely, $\frac{d}{d s}\left(\frac{\partial \mathcal{E}}{\partial \dot{r}^{m}}\right)=\frac{\partial \mathcal{E}}{\partial r^{m}}$. The paths minimizing $\int d s \mathcal{E}$, which 


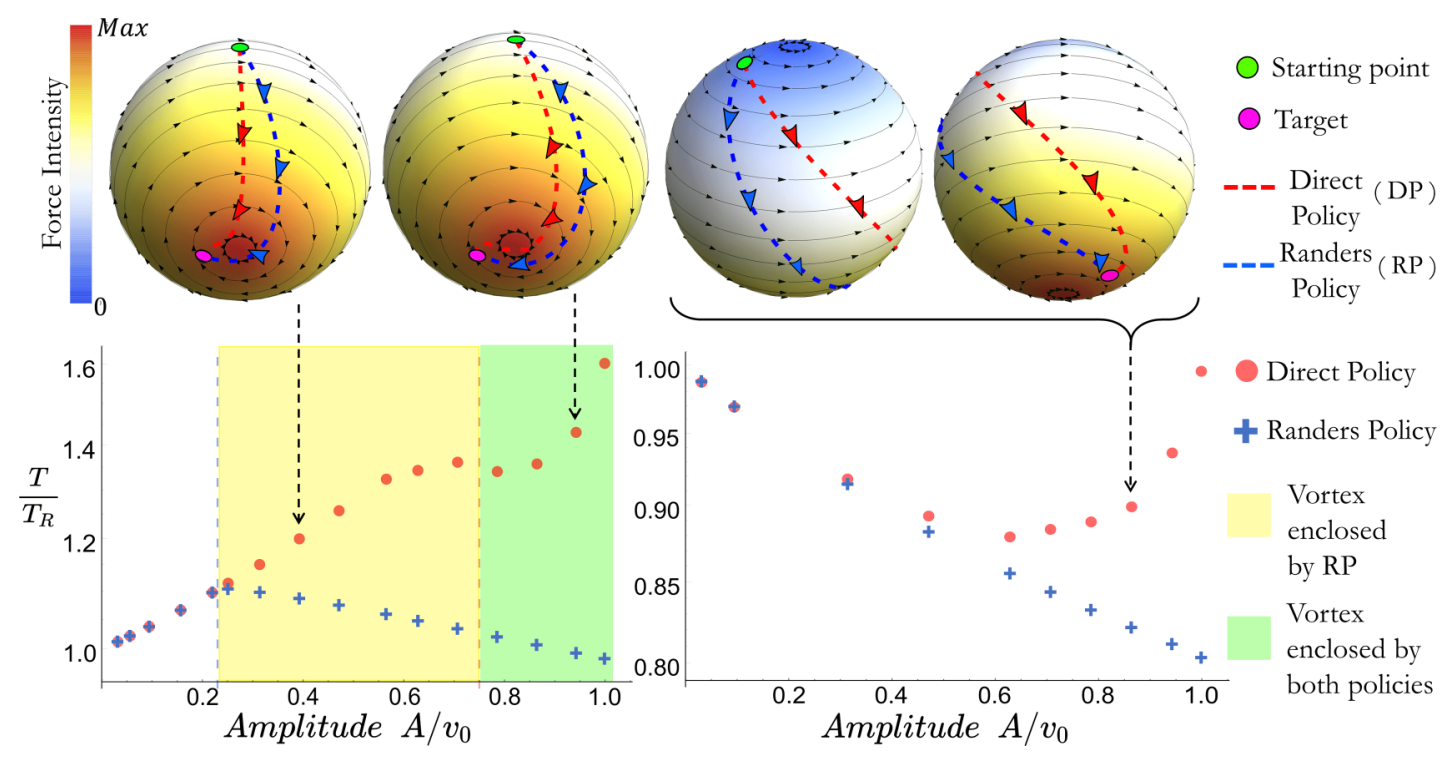

FIG. 2. Comparison between the Randers policy (RP, blue crosses) and the direct policy (DP, red circles) in terms of the arrival times $T$ in units of $T_{R}$ - the optimal navigation time in the absence of $\boldsymbol{f}$-as a function of the force amplitude. For both strategies, the target counts as reached when the microswimmer enters a region of radius $\epsilon=0.01$. On the left: analysis of the paths connecting the points $\left(\theta_{0}, \phi_{0}\right)=\left(\frac{\pi}{2}, 0\right) \rightarrow$ $\left(\theta_{1}, \phi_{1}\right)=\left(\frac{11 \pi}{12}, \frac{14 \pi}{10}\right)$. On the right: study of the paths linking two diametrically opposite points $\left(\theta_{0}, \phi_{0}\right)=\left(\frac{\pi}{8}, 0\right) \rightarrow\left(\theta_{1}, \phi_{1}\right)=\left(\frac{7 \pi}{8}, \pi\right)$. In either case the DP is suboptimal and there is a clear gain in following the RP, especially when the force is stronger. In the upper part of the figure there are three different exemplar trajectories for given values of the force amplitude as indicated by the dashed black arrow. The dashed lines on every sphere represent the paths for each navigation strategy (RP: blue; DP: red) and the arrows on top of them show the corresponding heading direction of the microswimmer. The gradient on each sphere indicates the force intensity, while the solid black arrows represent its direction.

also minimize the travel time $T$, satisfy the Randers metric geodesic equation

$$
\ddot{r}^{k}+\Gamma_{i j}^{k} \dot{r}^{i} \dot{r}^{j}=0
$$

where the Christoffel symbol $\Gamma_{i j}^{k}$ is defined via $\Gamma_{i j}^{k} \equiv$ $\frac{1}{2} g^{k m}\left(g_{i m, j}+g_{j m, i}-g_{i j, m}\right)$, with $g^{k m}$ being the inverse of the fundamental tensor defined in (4) and $g_{i j, m} \equiv \partial_{m} g_{i j}$. Thus, the solutions of the geodesic equation (6) provide optimal navigation paths for a microswimmer moving in the presence of the force field $\boldsymbol{f}$ on a generic Riemannian manifold $\mathcal{M}$. Notably, this formalism has the advantage of being sufficiently compact and elegant to enable the analysis of complex scenarios as well as possible generalizations. In what follows, we apply these theoretical concepts to the case in which the motion takes place on a sphere.

We can now directly compare the forward and backward paths in this setup, by showing how the area of the portion of sphere enclosed in the forward-backward loop varies with the intensity of the external force, A. In Fig. 1(b), we show the results obtained for one choice of initial and final points. The area enclosed in the loop grows as the intensity of the force increases, which is expected since both paths deviate more from the Riemannian geodesic (the optimal path in the absence of external force). Interestingly, the enclosed area undergoes a jump when the vortex at the south pole is encircled, as beyond a certain threshold in the force intensity the microswimmer can exploit the vortex to reach the goal more quickly and this causes an abrupt change in the shape of the optimal forward path. The scaling with $A$ is affected by this change, going from being essentially linear (black dashed line) to sublinear.

\section{PERFORMANCE ASSESSMENT}

We can now analyze the optimal paths obtained by following the Finsler geometry-based approach, which we call the Randers policy (RP), in comparison with a benchmark, which we refer to as the direct policy (DP), in which the microswimmer always points in the direction of the target, regardless of the force field [21]. To this end, we compute the time $T$ required to reach the target in units of the time $T_{R}$ it would take in the absence of any external force, as a function of the maximum force on the sphere.

\section{A. Optimal navigation on a sphere}

Let us first consider a sphere of radius unity embedded in $\mathbb{R}^{3}$. The position of the microswimmer on this surface can be written in spherical coordinates as $\boldsymbol{r}=(\theta, \phi)$. The corresponding Riemannian metric $h$ in spherical coordinates has the components $h_{\theta \theta}=1, h_{\phi \phi}=\sin ^{2} \theta$, and $h_{\theta \phi}=h_{\phi \theta}=0$. The force field is then $\boldsymbol{f}(\boldsymbol{r})=f_{\theta}(\theta, \phi) \hat{\boldsymbol{e}}_{\theta}+f_{\phi}(\theta, \phi) \hat{\boldsymbol{e}}_{\phi}$.

\section{Force field with two vortices}

As a first example, we choose $f_{\theta}(\theta, \phi)=0$ and $f_{\phi}(\theta, \phi)=$ $\frac{A \theta}{\pi \sin \theta}$, where $A$ sets the amplitude of the field, which is constrained as $A<v_{0}$. This divergence-free force field is characterized by a pair of vortices at the poles of the sphere and its intensity is maximum (minimum) at the south (north) pole. We can then write the explicit expression of the Randers metrics $\mathcal{L}$ in our case as follows:

$$
\mathcal{L}=\frac{\sqrt{v_{0}^{2} \sin ^{2} \theta \dot{\phi}^{2}+\left(v_{0}^{2}-A^{2} \theta^{2} / \pi^{2}\right) \dot{\theta}^{2}}-A \dot{\phi} \theta \sin \theta / \pi}{v_{0}^{2}-A^{2} \theta^{2} / \pi^{2}} .
$$



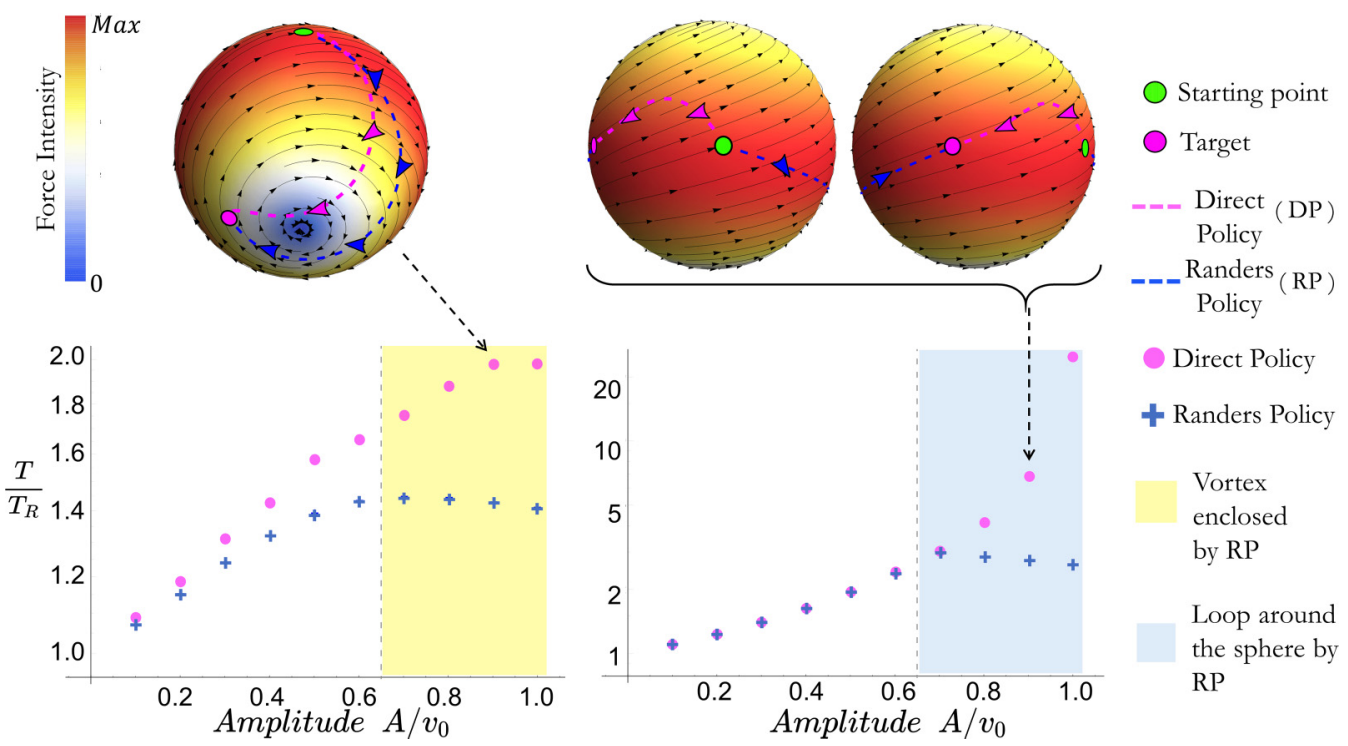

FIG. 3. Comparison between the arrival times of the Randers policy (RP, blue crosses) and the direct policy (DP, magenta circles) as a function of the maximum force intensity on the sphere. On the left: analysis of the paths connecting the points $\left(\theta_{0}, \phi_{0}\right)=\left(\frac{\pi}{2}, 0\right) \rightarrow\left(\theta_{1}, \phi_{1}\right)=$ $\left(\frac{5 \pi}{6}, \frac{3 \pi}{2}\right)$. On the right: study of the paths linking the points $\left(\theta_{0}, \phi_{0}\right)=\left(\frac{\pi}{2}, 0\right) \rightarrow\left(\theta_{1}, \phi_{1}\right)=\left(\frac{\pi}{2}, \frac{5 \pi}{3}\right)$. In either case the DP always takes longer than the RP, which gets more advantageous the stronger the force. In the upper part of the figure there are two examples of how the trajectories look for a given value of the force as indicated by the dashed black arrow.

It is then possible to determine the fundamental tensor $g_{i j}$, the relative Christoffel symbols $\Gamma_{i j}^{k}$, and the corresponding geodesic equations using their definitions in (4) and (6). We further choose the following initial conditions: $\theta(0)=\theta_{0}$, $\phi(0)=\phi_{0}, \dot{\theta}(0)=-\sin \varphi_{0}+f_{\theta}\left(\theta_{0}, \phi_{0}\right)$, and $\dot{\phi}(0)=\frac{\cos \varphi_{0}}{\sin \theta_{0}}+$ $f_{\phi}\left(\theta_{0}, \phi_{0}\right)$. Here, $\left(\theta_{0}, \phi_{0}\right)$ is the starting position while $\varphi_{0}$ represents the initial heading direction of the microswimmer (measured counterclockwise with respect to the $\hat{\boldsymbol{e}}_{\phi}$ direction), which we scan when using the shooting method, selecting the one that takes the shortest time. Moreover, we parametrize the trajectory using the proper time of the microswimmer (i.e., we set $s=\tau$ ), which implies that $\mathcal{L}$ will be a conserved quantity along these paths. A clear advantage of using this parametrization is that it is independent of the choice of a coordinate system and does not impose any restrictions on the shape of the trajectories.

In Fig. 2 we show the results obtained for two different choices of the initial and final points. In either case, for small values of the force, the two strategies do not show substantial differences in terms of performance. However, for the example shown on the left in Fig. 2, two particular situations can be observed. For larger values of the force (yellow and green regions) the RP (blue crosses) exploits the presence of the vortex at the south pole and at the same time the relative gain with respect to the DP (red circles) grows. In fact, following the former strategy makes it possible for the microswimmer to take up to $40 \%$ less time to reach the target. Moreover, for sufficiently large values of the force intensity (green region), the DP also includes the vortex. This slightly helps the swimmer, although just for a small range of values (see the local minimum in the green region). In addition, the relative gain following the RP is substantial (up to about $20 \%$ in terms of arrival time) even when this strategy does not imply the exploitation of any specific force field structures (see the plot on the right in Fig. 2).

\section{Force field with a sink and a spiral}

As a second example, let us consider a more complicated force field described by $f_{\theta}(\theta, \phi)=\frac{A}{\sqrt{\mu}}(\theta-\pi)$ and $f_{\phi}(\theta, \phi)=\frac{A}{\sqrt{\mu}}(\theta+\pi)$, where we have defined $\mu=$ $\max _{\theta}\left[\sin ^{2} \theta(\theta+\pi)^{2}+(\theta-\pi)^{2}\right]$, so that $A$ sets the force amplitude, which is then constrained as $A<v_{0}$. This force field is characterized by a singular point (sink) at the north pole and a spiral with null intensity in its center at the south pole.

The expression of the corresponding Randers metrics $\mathcal{L}$ [using its definition in (3)] is a bit more involved:

$$
\mathcal{L}=\frac{\sqrt{\left[v_{0}^{2}-\frac{A^{2}}{\mu}(\theta-\pi)^{2}\right] \sin ^{2} \theta \dot{\phi}^{2}+\left[v_{0}^{2}-\frac{A^{2}}{\mu} \sin ^{2} \theta(\theta+\pi)^{2}\right] \dot{\theta}^{2}+2 \frac{A^{2}}{\mu} \sin ^{2} \theta\left(\theta^{2}-\pi^{2}\right) \dot{\phi} \dot{\theta}}-\frac{A}{\sqrt{\mu}}\left[\sin ^{2} \theta(\theta+\pi) \dot{\phi}+(\theta-\pi) \dot{\theta}\right]}{v_{0}^{2}-\frac{A^{2}}{\mu}\left[\sin ^{2} \theta(\theta+\pi)^{2}+(\theta-\pi)^{2}\right]} .
$$

Then, once again we can determine the fundamental tensor $g_{i j}$, the Christoffel symbols $\Gamma_{i j}^{k}$, and the corresponding geodesic equations simply using their definitions (4) and (6). Finally, we shall equip the obtained ordinary differential equation (ODE) system with the proper initial conditions and solve the navigation problem.
Notably, also with this choice of the force, we observe that there is always a clear gain in following the Randers policy. The comparison with the performance achieved using the direct policy is shown in Fig. 3 where the displayed results correspond to two different choices of the target position. 
Concerning the example on the left in Fig. 3, you can see how exploiting the presence of the spiral allows the swimmer following the RP to take up to $30 \%$ less time to reach the target when compared to the DP.

Remarkably, the relative gain achieved by following the RP becomes extremely more significant (up to about $85 \%$ in terms of arrival time) when the RP implies the swimmer makes a full turn around the sphere, as you can see from the example on the right in Fig. 3 (blue region). This large difference between the two strategies is also due to the fact that following the DP entails the swimmer having to point against the force direction where it is most intense (on the equator), making this policy highly disadvantageous.

\section{B. Optimal navigation on a torus}

Let us now show how one can use the same formalism to address the problem of optimal navigation in a different geometry. The motion takes place on a torus and the position of the microswimmer on its surface can be defined by

$$
\begin{aligned}
x(\theta, \phi) & =(R+r \cos \theta) \cos \phi, \\
y(\theta, \phi) & =(R+r \cos \theta) \sin \phi, \\
z(\theta, \phi) & =r \sin \theta,
\end{aligned}
$$

in terms of the angles $\theta$ and $\phi$; respectively, the poloidal and toroidal directions (see Fig. 4). Let us fix the two characteristic radii of the torus to be $R=2$ and $r=1$ without loss of generality. The corresponding Riemannian metric $h$ has the

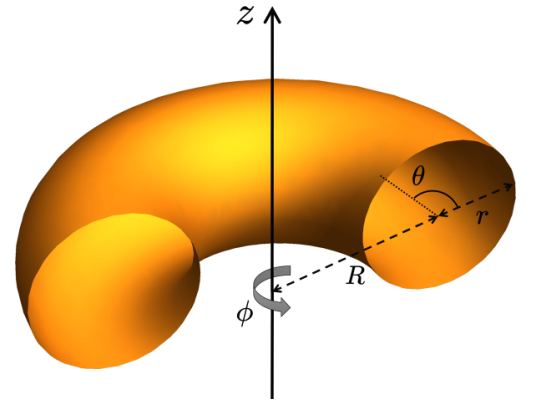

FIG. 4. Scheme of the parametrization of a toroidal surface.

components $h_{\theta \theta}=1, h_{\phi \phi}=(2+\cos \theta)^{2}$, and $h_{\theta \phi}=h_{\phi \theta}=0$. The force field is then $\boldsymbol{f}(\theta, \phi)=f_{\theta}(\theta, \phi) \hat{\boldsymbol{e}}_{\theta}+f_{\phi}(\theta, \phi) \hat{\boldsymbol{e}}_{\phi}$. For the sake of illustration, we consider a force field described by the following equations:

$$
\begin{aligned}
f_{\theta}(\theta, \phi) & =\frac{A}{\sqrt{2}} \sin \phi, \\
f_{\phi}(\theta, \phi) & =\frac{A}{\sqrt{2}} \frac{\cos \theta}{(2+\cos \theta)},
\end{aligned}
$$

where $A$ sets its amplitude, which is constrained as $A<v_{0}$. This force field is characterized by a pair of vortices and another of saddle points with null intensity in their centers.

We can now write the expression of the Randers metrics $\mathcal{L}$ in this new case. Using the definitions in (3) and (7), we get to

$$
\mathcal{L}=\frac{\sqrt{(2+\cos \theta)^{2}\left(v_{0}^{2}-\frac{A^{2}}{2} \sin ^{2} \theta\right) \dot{\phi}^{2}+\left(v_{0}^{2}-\frac{A^{2}}{2} \cos ^{2} \theta\right) \dot{\theta}^{2}+A^{2} \sin \phi \cos \theta(2+\cos \theta) \dot{\phi} \dot{\theta}}-\frac{A}{\sqrt{2}}[\cos \theta(2+\cos \theta) \dot{\phi}+\sin \phi \dot{\theta}]}{v_{0}^{2}-\frac{A^{2}}{2}\left(\cos ^{2} \theta+\sin ^{2} \phi\right)} .
$$

As already done for the sphere, it is then possible to determine the fundamental tensor $g_{i j}$, the Christoffel symbols $\Gamma_{i j}^{k}$, and the corresponding geodesic equations using their definitions reported in the previous section.

We further choose the following initial conditions: $\theta(0)=$ $\theta_{0}, \phi(0)=\phi_{0}, \dot{\theta}(0)=\sin \varphi_{0}+f_{\theta}\left(\theta_{0}, \phi_{0}\right), \dot{\phi}(0)=\frac{\cos \varphi_{0}}{\left(2+\cos \theta_{0}\right)}+$ $f_{\phi}\left(\theta_{0}, \phi_{0}\right)$, where the initial heading direction of the microswimmer $\varphi_{0}$ is measured counterclockwise with respect to the $\hat{\phi}$ direction. Once again, we find the time-optimal paths by means of a shooting method and parametrize the microswimmer trajectories using its proper time, i.e., setting $s=\tau$.

We can now directly compare the performance achieved with the Randers policy (RP) with that of the direct policy (DP). In Fig. 5 we show the analysis of the comparison between the arrival times obtained for two different choices of the initial and final points on the torus.

In both cases, the swimmer following the RP has an increasing gain as the intensity of the force grows, confirming what has already been observed on the sphere. For the example shown on the left in Fig. 5, you can see how exploiting the presence of the vortex allows the swimmer which follows the RP to reach the target faster (yellow and green regions). In fact, following this strategy makes it possible for the mi- croswimmer to take up to $20 \%$ less time when compared to the DP. Moreover, for sufficiently large values of the force intensity (green region), the DP also includes the vortex, slightly helping the swimmer.

In addition, the relative gain achieved by following the $\mathrm{RP}$ is even more significant (up to about $70 \%$ in terms of arrival time) when the RP strategy implies the exploitation of the topological properties of the surface, as you can see from the example on the right in Fig. 5 (blue region).

\section{ISOCHRONE ANALYSIS}

To study more generally the behavior and the shape of the optimal trajectories coming from the RP, we analyze the so-called isochrones in the illustrative examples of the sphere introduced in Sec. IV A. These are curves of equal travel time obtained by fixing the microswimmer initial position $\left(\theta_{0}, \phi_{0}\right)$ and varying the starting angle $\varphi_{0}$ from 0 to $2 \pi$. They can be seen as one-dimensional wave fronts of microswimmers that propagate onto the sphere following the Randers geodesics (6). 


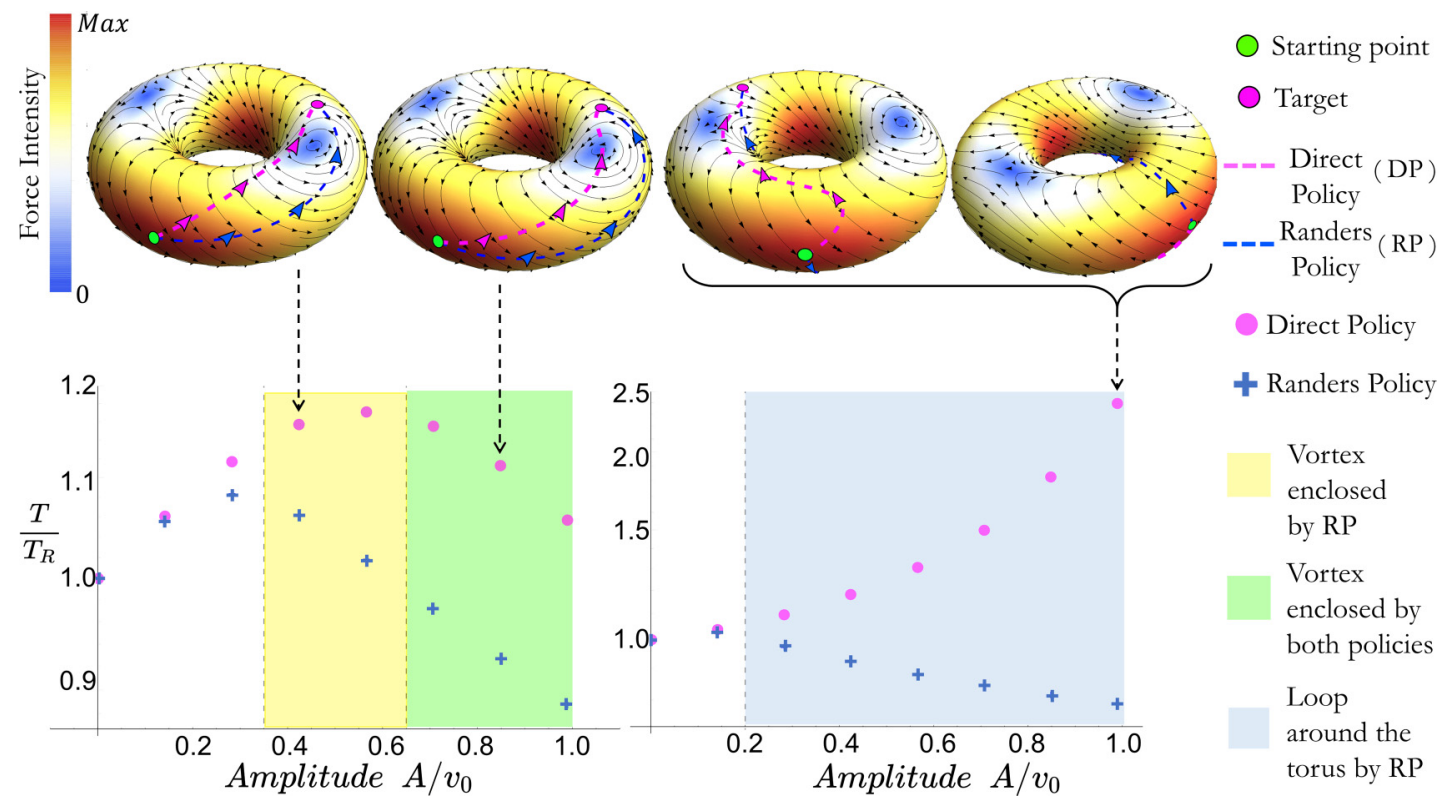

FIG. 5. Performance comparison between the Randers policy (RP, blue crosses) and the direct policy (DP, magenta circles). For both strategies, the target counts as reached when the microswimmer enters a region of radius $\epsilon=0.05$. On the left: analysis of the paths connecting the points $\left(\theta_{0}, \phi_{0}\right)=\left(0, \frac{3 \pi}{2}\right) \rightarrow\left(\theta_{1}, \phi_{1}\right)=\left(\frac{\pi}{2}, \frac{\pi}{4}\right)$. On the right: study of the paths linking the points $\left(\theta_{0}, \phi_{0}\right)=\left(0, \frac{3 \pi}{2}\right) \rightarrow\left(\theta_{1}, \phi_{1}\right)=\left(\frac{\pi}{2}, \frac{3 \pi}{4}\right)$. In either case there is a clear gain in following the RP, especially when the force is stronger. In the upper part of the figure there are three different examples of how the paths look for a given value of the force as indicated by the dashed black arrow. The dashed lines on every sphere represent the paths for each navigation strategy (RP: blue; DP: magenta) and the arrows on top of them show the corresponding heading direction of the microswimmer. The gradient on each sphere indicates the force intensity, while the solid black arrows represent its direction.

\section{A. Force field with two vortices}

In Fig. 6(a) we show some isochrones (solid lines) corresponding to the optimal paths starting from a point on the equator (green circle), in the presence of the force field introduced in Sec. IV A 1 with $A=\frac{1}{2} v_{0}$.

We observe that isochrones can feature self-intersections [see the example at $\tau=2.9 v_{0}^{-1}$ on the right in Fig. 6(a)]. These are spots on the sphere for which there are multiple solutions to the problem of optimal navigation. Moreover, the isochrones can develop cusps, as highlighted in Fig. 6(a) (see Supplemental Material [40], movie), which are points at which neighboring geodesics meet. These cusp are analogs of conjugate points in general relativity [41], and related to the caustics in optics, as they represent domains on the isochrones with a higher density of geodesics [42]. Remarkably, these self-intersections and cusps occur only in the presence of
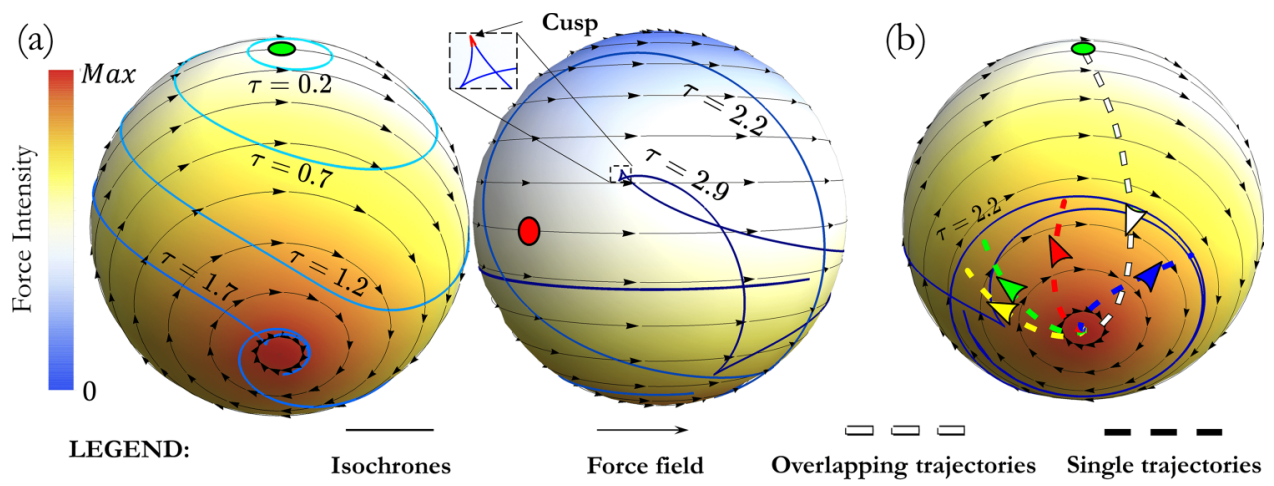

FIG. 6. Analysis of the isochrones starting from the point $\left(\theta_{0}, \phi_{0}\right)=(\pi / 2,0)$ (green circle) in the presence of the force field characterized by two vortices with $A=\frac{1}{2} v_{0}$. The red circle indicates the point diametrically opposite to the starting point and provides a guide to the eye. The color code on each sphere shows the force intensity from small (blue) to high (red), while the black arrows represent its direction. The time $\tau$ is reported in units of $v_{0}^{-1}$. (a) Isochrones (solid lines) at six different times. On the left: view of a region of stronger force. On the right: front view with an enlargement of a cusp highlighted in red, a point with a high density of geodesics. Also, notice the presence of self-intersections, points where two optimal paths collide. (b) Representation of four specific paths (dashed lines) passing close to the center of the vortex at the south pole. The arrows on top of them show the corresponding heading direction of the microswimmer and their color refers to the starting angle (green: $\varphi_{0}=4.7122$; blue: $\varphi_{0}=4.7123$; red: $\varphi_{0}=4.7124$; yellow: $\varphi_{0}=4.7125$ ). In the initial part where they overlap, the path is highlighted in white. This shows a strong dependence on initial conditions for optimal trajectories passing close to the vortex at the south pole. 

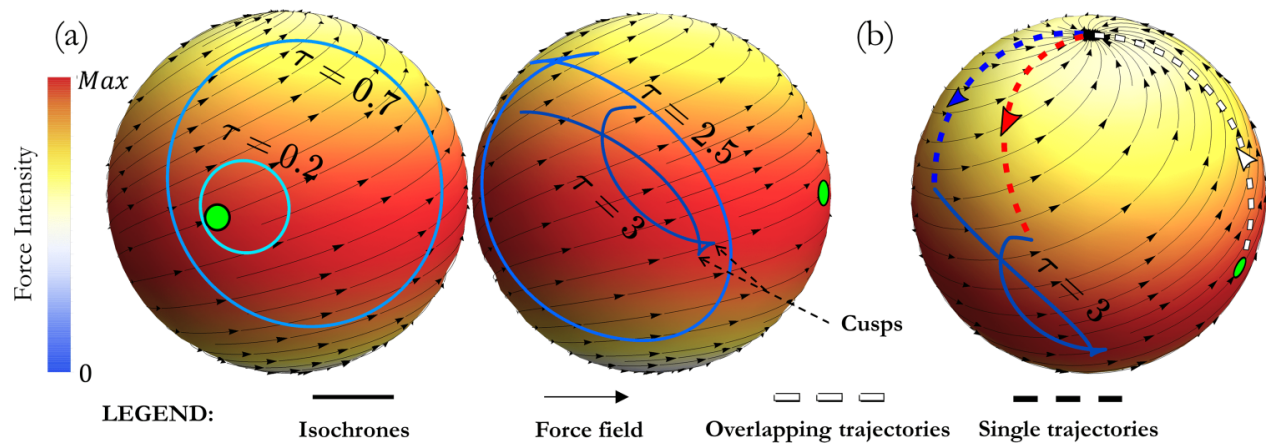

FIG. 7. Isochrones (solid blue lines) at four different times starting from the point $\left(\theta_{0}, \phi_{0}\right)=(\pi / 2,0)$ (green circle) in the presence of the force field introduced in Sec. IV A 2 with $A=0.7 v_{0}$. (a) On the left: front view. On the right: side view, where the two isochrones display self-intersections, cusps, and a discontinuity due to the presence of the singularity at the north pole. (b) Representation of two specific paths (dashed lines) passing close to the singularity at the north pole. The arrows on top of them show the corresponding heading direction of the microswimmer and their color refers to the starting angle (red: $\varphi_{0}=1.5707$; blue: $\left.\varphi_{0}=1.5708\right)$. In the initial part where they overlap, the path is highlighted in white.

a force field, as otherwise the isochrones on a sphere form concentric circles.

The isochrones are considerably distorted after they encounter the vortex at the south pole [see the isochrone at $\tau=1.7 v_{0}^{-1}$ in Fig. 6(a)]. The meaning of such deformation can be understood by looking at Fig. 6(b). Here are shown four optimal trajectories (dashed lines) starting from a point on the equator (green circle) and ending at time $\tau=2.2 v_{0}^{-1}$ on the corresponding isochrone (blue solid line). Their initial angles $\varphi_{0}$ differ only by $\Delta \varphi_{0}=10^{-4}$. Such paths initially overlap (white dashed line) and separate only once they reach the south pole. The observed strong dependence on initial conditions provides an interesting insight if one thinks of natural extensions of the model, e.g., the introduction of rotational noise. A direct implication of such abrupt nonlinear effects is indeed that, in the presence of a singularity in the force field, noise cannot be perturbatively included in the model and one must therefore consider alternative approaches [43].

\section{B. Force field with a sink and a spiral}

In Fig. 7 we show the isochrones (solid lines) corresponding to the optimal paths starting from a point on the equator (green circle), in the presence of the force field introduced in Sec. IV A 2 with $A=0.7 v_{0}$.

Once again we find that there are spots on the sphere for which there are multiple solutions to the problem of optimal navigation (self-intersections) and others with a higher density of geodesics (cusps) [see the example at $\tau=3 v_{0}^{-1}$ on the right in Fig. 7(a)].

However, here we can see a new peculiar feature of isochrones that has not been observed in the case illustrated above: they can be discontinuous (e.g., see the isochrones at $\tau=2.5 v_{0}^{-1}$ and $\tau=3 v_{0}^{-1}$ ). This is certainly due to the presence of the singularity (sink) at the north pole and can be better understood by looking at Fig. 7(b). Here are shown two optimal trajectories (dashed lines) starting from the point on the equator and ending at time $\tau=3 v_{0}^{-1}$ on the corresponding isochrone (green solid line). Their initial angles $\varphi_{0}$ differ only by $\Delta \varphi_{0}=10^{-4}$. Such paths initially overlap (white dashed line) and separate only once they reach the north pole. Intu- itively, since in its proximity there is an abrupt change in the force field direction, even a very small difference in the microswimmer position and orientation can lead to very different future scenarios. This prevents the use of perturbative methods to introduce noise into the model for trajectories passing in the neighborhood of a singularity, as already pointed out above.

\section{CONCLUDING REMARKS}

We formulate and discuss a geometric description of the optimal navigation problem for microswimmers on curved manifolds. We show that this problem can be solved by finding the geodesics of a nonreversible Finsler metric of Randers type, providing a link between microswimmers physics and generalizations of general relativity. Our proposed geometric approach provides tools for solving the optimal navigation problem in as yet unexplored, as well as more complex scenarios, such as paths for microswimmers escaping from harmful regions [44]. Here, we assumed to have full control over the microswimmer heading direction. However, our results could still be used to deal with rotational diffusion. We can indeed continuously adjust the orientation of the microswimmer to make it stay close to the optimal path of the noise-free system. Another assumption we made is that of stationary flow. Although this constraint cannot be relaxed as is necessary to keep the analogy with Randers spaces and compute the corresponding geodesic equation (6), our results still hold true as long as there is a separation of timescales, with the characteristic timescale of the flow being much larger than the navigation time.

Further research might use this analytical approach to benchmark numerical optimization procedures relying on machine learning algorithms [20,21,45] and should generalize it by including noise in the model. It would also be interesting to relax the constraint on the force amplitude, possibly leading to the study of both timelike and spacelike trajectories [46]. Finally, one can study how microswimmers can sense curvature by considering finite-size swimmers able to estimate spatial derivatives and the metric $[47,48]$. 


\section{ACKNOWLEDGMENTS}

L.P. acknowledges A. Codutti for fruitful discussions. This work was supported by the Max Planck Society.

\section{APPENDIX A: DETAILS ON THE ANALYTICAL DERIVATION OF THE RANDERS METRIC}

The aim of this section is to show all the calculations leading to the expression of the Randers metric $\mathcal{L}$.

First of all, we have to rewrite the equation of motion of the microswimmer in a covariant form:

$$
\dot{r}^{i} v=v_{0}^{i}+f^{i},
$$

where $\dot{r}^{i}=\frac{d r^{i}}{d s}$ and $v=\frac{d s}{d \tau}$. If we now isolate $v_{0}^{i}$, square both sides of the equation and then multiply them by the metric tensor $h$, we get to the following expression:

$$
h_{i j}\left(\dot{r}^{i} v-f^{i}\right)\left(\dot{r}^{j} v-f^{j}\right)=h_{i j} v_{0}^{i} v_{0}^{j} .
$$

Notice that the rhs represents the self-propelling speed of the microswimmer. Therefore, we can solve this equation for $v$ and obtain:

$$
v=\frac{ \pm \sqrt{h_{i j} \dot{r}^{i} \dot{r}^{j} h_{l m}\left(v_{0}^{l} v_{0}^{m}-f^{l} f^{m}\right)+\left(h_{i j} \dot{r}^{i} f^{j}\right)^{2}}+h_{i j} \dot{r}^{i} f^{j}}{h_{i j} \dot{r}^{i} \dot{r}^{j}} .
$$

We can now select just the solution with the + in front of the square root. Indeed, for $\boldsymbol{f}=0$ we correctly obtain $v>0$, while the other solution would give $v<0$.

Lastly, we can get the desired expression for the Randers metric $\mathcal{L}$ by simply computing the reciprocal of $v$ :

$$
\mathcal{L}=\frac{h_{i j} \dot{r}^{i} \dot{r}^{j}}{\sqrt{h_{i j} \dot{r}^{i} \dot{r}^{j} h_{l m}\left(v_{0}^{l} v_{0}^{m}-f^{l} f^{m}\right)+\left(h_{i j} \dot{r}^{i} f^{j}\right)^{2}}+h_{i j} \dot{r}^{i} f^{j}},
$$

and then multiplying both the numerator and denominator by $\sqrt{h_{i j} \dot{r}^{i} \dot{r}^{j} h_{l m}\left(v_{0}^{l} v_{0}^{m}-f^{l} f^{m}\right)+\left(h_{i j} \dot{r}^{i} f^{j}\right)^{2}}-h_{i j} \dot{r}^{i} f^{j}$, to get the final expression:

$$
\mathcal{L}=\frac{\sqrt{h_{i j} \dot{r}^{i} \dot{r}^{j} h_{l m}\left(v_{0}^{l} v_{0}^{m}-f^{l} f^{m}\right)+\left(h_{i j} \dot{r}^{i} f^{j}\right)^{2}}-h_{i j} \dot{r}^{i} f^{j}}{h_{i j}\left(v_{0}^{i} v_{0}^{j}-f^{i} f^{j}\right)} .
$$

To simplify the latter expression, we can define some new quantities (using the same notation as in the main text): $v_{0}^{2} \equiv$ $h_{i j} v_{0}^{i} v_{0}^{j}, \lambda^{-1} \equiv v_{0}^{2}-h_{i j} f^{i} f^{j}, a_{i j} \equiv h_{i j} \lambda+f_{i} f_{j} \lambda^{2}, b_{i} \equiv-f_{i} \lambda$. This leads us to:

$$
\mathcal{L}=\sqrt{a_{i j} \dot{r}^{i} \dot{r}^{j}}+b_{i} \dot{r}^{i},
$$

which is the sought compact expression of the Randers metric $\mathcal{L}$.

ing electric and magnetic fields, Soft Matter 14, 4741 (2018).

[13] K. Villa, J. Viktorova, J. Plutnar, T. Ruml, L. Hoang, and M. Pumera, Chemical microrobots as self-propelled microbrushes against dental biofilm, Cell Rep. Phys. Sci. 1, 100181 (2020).

[14] H. C. Berg, E. Coli in Motion (Springer Science and Business Media, New York, 2008).

[15] D. Bray, Cell Movements (Garland Science, New York, 2000).

[16] R. R. Bennett and R. Golestanian, A steering mechanism for phototaxis in Chlamydomonas, J. R. Soc., Interface $\mathbf{1 2}$ 20141164 (2015).

[17] G. Gompper, R. G. Winkler, T. Speck, A. Solon, C. Nardini, F. Peruani, H. Löwen, R. Golestanian, U. B. Kaupp, L. Alvarez et al., The 2020 motile active matter roadmap, J. Phys.: Condens. Matter 32, 193001 (2020).

[18] E. Zermelo, ZAMM - J. Applied Math. Mechanics 11, 114 (1931).

[19] B. Liebchen and H. Löwen, Optimal navigation strategies for active particles, Europhys. Lett. 127, 34003 (2019).

[20] S. Colabrese, K. Gustavsson, A. Celani, and L. Biferale, Flow Navigation by Smart Microswimmers Via Reinforcement Learning, Phys. Rev. Lett. 118, 158004 (2017).

[21] L. Biferale, F. Bonaccorso, M. Buzzicotti, P. C. D. Leoni, and K. Gustavsson, Chaos: An interdisciplinary journal of nonlinear science, Chaos 29, 103138 (2019).

[22] E. Schneider and H. Stark, Optimal steering of a smart active particle, Europhys. Lett. 127, 64003 (2019).

[23] P. Castro-Villarreal and F. J. Sevilla, Active motion on curved surfaces, Phys. Rev. E 97, 052605 (2018).

[24] S. J. Callens, R. J. Uyttendaele, L. E. Fratila-Apachitei, and A. A. Zadpoor, Substrate curvature as a cue to guide spatiotem- 
poral cell and tissue organization, Biomaterials 232, 119739 (2020).

[25] S.-Z. Lin, Y. Li, J. Ji, B. Li, and X.-Q. Feng, Collective dynamics of coherent motile cells on curved surfaces, Soft Matter 16, 2941 (2020).

[26] S. Ehrig, J. Ferracci, R. Weinkamer, and J. W. C. Dunlop, Curvature-controlled defect dynamics in active systems, Phys. Rev. E 95, 062609 (2017).

[27] S. Henkes, M. C. Marchetti, and R. Sknepnek, Dynamical patterns in nematic active matter on a sphere, Phys. Rev. E 97, 042605 (2018).

[28] Z. Shen, Finsler Metrics with $\mathrm{K}=0$ and $\mathrm{S}=0$, Canadian J. Math. 55, 112 (2003).

[29] D. Bao, C. Robles, and Z. Shen, J. Diff. Geom. 66, 377 (2004).

[30] G. Randers, On an asymmetrical metric in the four-space of general relativity, Phys. Rev. 59, 195 (1941).

[31] P. Finsler, Über kurven und flächen in allgemeinen räumen, dissertation, University of Göttingen, 1918.

[32] G. W. Gibbons, C. A. R. Herdeiro, C. M. Warnick, and M. C. Werner, Stationary metrics and optical Zermelo-RandersFinsler geometry, Phys. Rev. D 79, 044022 (2009).

[33] D. C. Brody and D. M. Meier, Solution to the Quantum Zermelo Navigation Problem, Phys. Rev. Lett. 114, 100502 (2015).

[34] R. Golestanian, M. R. H. Khajehpour, and R. Mansouri, A test theory of the local structure of spacetime: A Finslerian approach, Class. Quantum Grav. 12, 273 (1995).

[35] B. Schutz, Geometrical Methods of Mathematical Physics (Cambridge University Press, Cambridge, UK, 1980).

[36] D. Bao, S. S. Chern, and Z. Shen, An Introduction to RiemannFinsler Geometry (Springer, New York, 2000).
[37] S. S. Chern and Z. Shen, Riemann-Finsler Geometry (World Scientific, Singapore 2005).

[38] X. Cheng and Z. Shen, Finsler Geometry: An Approach Via Randers Spaces (Springer, New York, 2012).

[39] J. M. Lee, Riemannian geodesics, in Riemannian Manifolds: An Introduction to Curvature (Springer, New York, 1997), pp. 65-89.

[40] See Supplemental Material at http://link.aps.org/supplemental/ 10.1103/PhysRevResearch.3.023125 for the movie that shows the degeneracy of the optimization problem at the cusps.

[41] Y. Manor, Caustics in general relativity I. The phase function, Ann. Phys. (NY) 106, 407 (1977).

[42] V. Arnold, Singularities of Caustics and Wave Fronts (Springer, Netherlands, 1990).

[43] E. Woillez, Y. Zhao, Y. Kafri, V. Lecomte, and J. Tailleur, Activated Escape of a Self-Propelled Particle from a Metastable State, Phys. Rev. Lett. 122, 258001 (2019).

[44] W.-W. Tso and J. Adler, Negative chemotaxis in Escherichia coli, J. Bacteriol. 118, 560 (1974).

[45] F. Cichos, K. Gustavsson, B. Mehlig, and G. Volpe, Machine learning for active matter, Nat. Mach. Intell. 2, 94 (2020).

[46] E. Caponio, M. A. Javaloyes, and M. Sánchez, On the interplay between Lorentzian Causality and Finsler metrics of Randers type, Rev. Mat. Iberoamericana 27, 919 (2011).

[47] P. Stavrinos, Generalized-Finslerian equation of geodesic deviations, Rep. Math. Phys. 32, 339 (1993).

[48] D. Bao and C. Robles, Ricci and flag curvatures in Finsler geometry, A Sampler of Riemann-Finsler Geometry (Cambridge University Press, Cambridge, UK, 2004), Vol. 50, p. 197. 